\title{
Case-Control Study of the Effects of Gut Microbiota Composition on Neurotransmitter Metabolic Pathways in Children With Attention Deficit Hyperactivity Disorder
}

\author{
Lin Wan ${ }^{1 \dagger}$, Wen-Rong Ge ${ }^{2 \dagger}$, Shan Zhang ${ }^{1}$, Yu-Lin Sun ${ }^{1}$, Bin Wang ${ }^{1}$ and Guang Yang ${ }^{1 *}$ \\ ${ }^{1}$ The First Medical Center of the Chinese PLA General Hospital, Beijing, China, ${ }^{2}$ Beijing Friendship Hospital, Capital Medical \\ University, Beijing, China
}

OPEN ACCESS

Edited by:

Andreas Martin Grabrucker University of Limerick, Ireland

Reviewed by:

Kiran Veer Sandhu,

University College Cork, Ireland

Silvia Turroni,

University of Bologna, Italy

${ }^{*}$ Correspondence:

Guang Yang

yangg301@sina.com

${ }^{\dagger}$ These authors have contributed equally to this work

Specialty section:

This article was submitted to Neuroendocrine Science,

a section of the journal

Frontiers in Neuroscience

Received: 16 October 2019

Accepted: 31 January 2020

Published: 18 February 2020

Citation:

Wan L, Ge W-R, Zhang S,

Sun Y-L, Wang B and Yang G (2020) Case-Control Study of the Effects

of Gut Microbiota Composition on Neurotransmitter Metabolic Pathways in Children With Attention Deficit

Hyperactivity Disorder.

Front. Neurosci. 14:127.

doi: 10.3389/fnins.2020.00127
Background: Attention-deficit/hyperactivity disorder (ADHD) is a neuropsychiatric condition that may be related to an imbalance of neural transmitters. The gut microbiota is the largest ecosystem in the human body, and the brain-gut axis theory proposes that the gut microbiome can affect brain function in multiple ways. The purpose of this study was to explore the gut microbiota in children with ADHD and assess the possible role of the gut microbiota in disease pathogenesis to open new avenues for ADHD treatment.

Methods: A case-control design was used. We enrolled 17 children aged 6-12 years with ADHD who were treated in the Pediatric Outpatient Department of the First Medical Center of the Chinese PLA General Hospital from January to June, 2019. Seventeen children aged 6-12 years were selected as the healthy control $(\mathrm{HC})$ group. Fecal samples of cases and controls were analyzed by shotgun metagenomics sequencing. Alpha diversity and the differences in the relative abundances of bacteria were compared between the two groups. Functional annotations were performed for the microbiota genes and metabolic pathways were analyzed using the Kyoto Encyclopedia of Genes and Genomes (KEGG).

Results: There was no significant difference in the alpha diversity of gut microbiota between the ADHD and HC groups. Compared with HCs, Faecalibacterium and Veillonellaceae were significantly reduced in children with $\operatorname{ADHD}(P<0.05)$, Odoribacter and Enterococcus were significantly increased [linear discriminant analysis (LDA) > 2]. At the species level, Faecalibacterium prausnitzii, Lachnospiraceae bacterium, and Ruminococcus gnavus were significantly reduced in the ADHD group $(P<0.05)$, while Bacteroides caccae, Odoribacter splanchnicus, Paraprevotella xylaniphila, and Veillonella parvula were increased $(P<0.05)$. Metabolic pathway analysis revealed significant between-group differences in the metabolic pathways of neurotransmitters (e.g., serotonin and dopamine) $(P<0.05)$. 
Conclusion: Composition differences of gut microbiota in subjects with ADHD may contribute to brain-gut axis alterations and affect neurotransmitter levels, which could contribute to ADHD symptoms.

Keywords: attention deficit hyperactivity disorder, child, gastrointestinal microbiome, shotgun metagenomics sequencing, neurotransmitter

\section{INTRODUCTION}

Attention-deficit/hyperactivity disorder (ADHD) is a neuropsychiatric disorder that occurs most frequently in school-age children and is characterized as inattention with or without excessive impulsivity and hyperactivity (Abramov et al., 2019). Previous studies have reported that ADHD pathogenesis may be associated with dysregulation of neurotransmitters such as dopamine, serotonin (5-hydroxytryptamine, 5-HT), and norepinephrine (Magula et al., 2019; Stewart et al., 2019; Suzuki et al., 2019). Others have shown that the incidence of ADHD may have a certain degree of heritability, and genes related to dopamine, norepinephrine, and 5-HT transmission have been found to be abnormally expressed in children with ADHD (Banerjee and Nandagopal, 2015; Karmakar et al., 2017; Kim et al., 2018). Although various theories have been proposed, the pathogenetic mechanisms underlying ADHD have not been fully clarified, which limits the development of new treatments.

Gut microbiota alterations may be associated with neurological conditions including Alzheimer's disease, epilepsy, and autism (Fan et al., 2019; Rude et al., 2019). Many researchers have proposed the existence of bidirectional regulation of the brain-gut axis, which involves gut microbiota metabolites that affect neurotransmitter levels, thereby influencing brain function (Melli et al., 2016; Khalil et al., 2019; Lacorte et al., 2019). In addition, nervous system activity can also impact gut microbiota composition. This bidirectional regulation is accomplished via complex neuroendocrine pathways (Khalil et al., 2019). The gut microbiota can adjust these pathways by regulating the levels of neurotransmitters and inflammatory factors and affecting the hypothalamic-pituitaryadrenal axis (Bermúdez-Humarán et al., 2019). Therefore, abnormal intestinal flora composition may lead to abnormal neurotransmitter secretion, which may promote the development of neuropsychiatric diseases.

We conducted a case-control study to analyze differences in intestinal flora composition between children with ADHD and healthy control (HC) children, explore ADHD pathogenesis, and investigate potential new treatments for ADHD.

\section{MATERIALS AND METHODS}

\section{Study Subjects}

Seventeen children aged 6-12 (median 8 years) with ADHD were selected from the Pediatric Outpatient Department of the First Medical Center of the PLA General Hospital between January and June, 2019. The inclusion criteria were: (1) The
Kiddie Schedule for Affective Disorders and Schizophrenia (K-SADS, Present and Lifetime Version scales) was used to diagnosis ADHD, and subjects met the diagnostic criteria for ADHD in the Diagnostic and Statistical Manual of Mental Disorders, Fifth Edition (DSM-5) (Ng et al., 2019) based on the opinion of an experienced child psychiatrist (GY or LW); (2) no history of respiratory or digestive tract infection within 1 month; (3) no use of probiotics within 1 month; (4) no history of digestive diseases or other chronic diseases; (5) body mass index (BMI) $<20 \mathrm{~kg} / \mathrm{m}^{2}$ (because obesity could cause gut microbiota abnormalities) (Salah et al., 2019); and (6) no allergic diseases such as allergic rhinitis or asthma. Seventeen children from different families aged 6-12 years (median 8 years) were selected as the HC group in the same period. The inclusion criteria were the same except that there was no diagnosis of ADHD based on DSM- 5 criteria by K-SADS. All of the participating children were born full-term with normal deliveries. Subjects were excluded if they were on a special diet (e.g., vegetarian). All parents of the participating children completed the Conners Parent Rating Scales (CPRS) to assess ADHD symptom severity and exclude subjects with depressive or anxiety symptoms. Participants maintained their regular dietary patterns for a week, and a food diary was recorded for participants from both groups during this period in order to exclude the potential influence of any changes in diet on the intestinal flora. Stool samples were collected at 8:00 am in the Pediatric Outpatient Department and stored in a sterile plastic cup at $-80^{\circ} \mathrm{C}$ prior to testing.

The study was approved by the PLA General Hospital Ethics Committee (no. 2018-278). All subjects' guardians were informed about the intentions of this study, and gave written informed consent was obtained in accordance with the Declaration of Helsinki.

\section{Sequencing and Analysis DNA Sequencing}

A total of 34 stool samples were collected from 17 ADHD patients and 17 age-matched HCs. We applied shotgun metagenomic sequencing to the whole genome of the microorganisms for each specimen. Bead beating was performed to rupture the bacteria, DNA was extracted with HiPure Stool DNA kits (Angen Biotech Co., Ltd., Guangzhou, China), and Qubit 4.0 software (Thermo Fisher Scientific, Waltham, MA, United States) was used for quality assessment. The library was prepared with a KAPA Hyper Prep Kit (KAPA Biosystems, Wilmington, MA, United States) and paired-end sequencing was performed on an Illumina NovaSeq platform (Illumina, San Diego, CA, United States) with a reading length of $150 \mathrm{bp}$ (PE150). 


\section{Species Abundance and Gene Function Annotations}

All genome sequencing data were preprocessed by KneadData ${ }^{1}$ to screen out low-quality short frame sequences and chimeric sequences among the structural primer sequences (Bolger et al., 2014). Bowtie2 (Langmead and Salzberg, 2012) was then used to align the reads with the human genome for host sequence contamination removal. This was carried out with human reference genome hg19) ${ }^{2}$.

HUMAnN2 (version v0.11.2) was used to analyze the species abundance, gene function, and metabolic pathways related to the processed sequencing data (Franzosa et al., 2018). HUMAnN2 first used MetaPhlAn2 (version 2.7.7, Li et al., 2014) to match the sequence with the established core genes to quickly locate the species included in the microbiota. Sequences were then compared with the pan-genome of the identified species and mapped to corresponding phylogenetic levels. The abundance of genes or gene families, and metabolic pathways were analyzed at different phylogenetic levels of interest.

To determine the gene functional annotations, we employed the Bowtie2 (version 2.3.4.3) to map the sequences after removing low-quality sequences and host sequences, to Integrated Gene Catalog databases and Kyoto Encyclopedia of Genes and Genomes (KEGG).On this basis, gene abundance and alpha diversity indexes were calculated, which involves using the Shannon, Chaol, and Simpson indexes to calculate the entropy values of gene abundance. Euclidean distance was also computed as the measurement of beta diversity, followed by principal component analysis (PCA) and permutational multivariate analysis of variance (PERMANOVA). PCA was performed using ade 4 package and PERMANOVA was carried out using vegan package ( $\mathrm{R}$ version 3.5 .3$)^{3}$.

\section{Bioinformatics Analysis}

Chi-square tests were performed by SPSS 21.0 to compare sex differences between the ADHD and HC groups, and independent-sample $t$-tests were used to compare age, BMI, and CPRS scores. Wilcoxon tests were used by SPSS 21.0 to assess differences in species abundance and gene function between the ADHD and HC groups. The LDA effect size (LEfSe) method was used to determine the most differentially abundant taxa at the genus and species levels between the two groups.

\section{RESULTS}

\section{Comparison of Clinical Data Between the ADHD and HC Groups}

A total of $17 \mathrm{ADHD}$ children were included in this study, including $14(82.3 \%)$ males and $3(17.7 \%)$ females with a median age of 8 (25th and 75th percentiles: 7,10$)$ and a mean BMI of $16.1 \pm 1.2 \mathrm{~kg} / \mathrm{m}^{2}$. The $17 \mathrm{HCs}$ included $13(76.5 \%)$ males and $4(23.5 \%)$ females with a median age of $8(7,9.5)$ and a mean

${ }^{1}$ https://bitbucket.org/biobakery/kneaddata

${ }^{2}$ http://hgdownload.soe.ucsc.edu/goldenPath/hg19/bigZips/hg19.fa.gz

${ }^{3}$ https://www.r-project.org/
BMI of $15.9 \pm 1.1 \mathrm{~kg} / \mathrm{m}^{2}$. There was no significant difference in the distributions of sex, age, or BMI between the two groups $(P>0.05)$. More children in the ADHD group $(12,70.5 \%)$ developed symptoms of constipation than in the HC group (2, $11.7 \%)$. The total CPRS scores were significantly different between the ADHD and $\mathrm{HC}$ groups ( $10.3 \pm 4.2$ vs. $2.2 \pm 0.63$, respectively; $P<0.05)$. There were no significant differences in the subscores for psychosomatic symptoms $(0.56 \pm 0.34$ vs. $0.53 \pm 0.41)$ or anxiety $(0.42 \pm 0.32$ vs. $0.51 \pm 0.35)(P>0.05$, Table 1$)$.

\section{Analysis of Intestinal Flora Diversity}

The Shannon $(9.67 \pm 0.42$ vs. $9.52 \pm 0.25)$, Chaol $(61.5 \pm 11.6$ vs. $57.5 \pm 9.8)$, and Simpson $(0.89 \pm 0.07$ vs. $0.88 \pm 0.06)$ indexes were calculated to assess the alpha diversity of fecal microbiota in the ADHD and HC groups. There were no significant differences in index values between the two groups (Figure 1A). At the genus level and the species level, PERMANOVA could not discriminate the ADHD from the HC group due to significant individual variation (Figures 1B,C).

\section{Analyses of Fecal Bacterial Community Abundance}

At the genus level, Wilcoxon tests showed that Faecalibacterium and Veillonellaceae were significantly reduced in the ADHD group, while Odoribacter was significantly higher $(P<0.05$, Figure 2A). The LEfSe results also indicated that Enterococcus was significantly increased in the ADHD group (LDA $>$ 2, Figure 2B).

At the species level, Wilcoxon tests showed that Faecalibacterium prausnitzii, Lachnospiraceae bacterium, and Ruminococcus gnavus were significantly decreased in the ADHD group, while Bacteroides caccae, Odoribacter splanchnicus, Paraprevotella xylaniphila, and Veillonella parvula were significantly increased $(P<0.05$, Figure 2C). The results of LEfSe showed that Odoribacteraceae and

TABLE 1 | Descriptive data of the ADHD and HC groups.

\begin{tabular}{|c|c|c|c|}
\hline & ADHD $(n=17)$ & $\mathrm{HC}(n=17)$ & $\boldsymbol{P}$ \\
\hline Sex, $n(\%)$ & & & 0.671 \\
\hline Male & $14(82.3 \%)$ & $13(76.5 \%)$ & \\
\hline Female & $3(15 \%)$ & $4(23.5 \%)$ & \\
\hline $\begin{array}{l}\text { Age, years; median (25th } \\
\text { and } 75 \text { th percentiles) }\end{array}$ & $8(7,10)$ & $8(7,9.5)$ & 0.701 \\
\hline BMI, mean (SD) & $16.1(1.2)$ & $15.9(1.1)$ & 0.652 \\
\hline Constipation, $n(\%)$ & 12 (70.5\%) & $2(11.7 \%)$ & $<0.05$ \\
\hline \multicolumn{4}{|c|}{ ADHD symptom severity, mean (SD) } \\
\hline Total CPRS score & $10.3(4.2)$ & $2.2(0.63)$ & $<0.05$ \\
\hline Conduct problems & $3.1(1.46)$ & $0.16(0.27)$ & $<0.05$ \\
\hline Impulsive-hyperactivity & $1.5(0.59)$ & $0.16(0.22)$ & $<0.05$ \\
\hline Hyperactivity & $3.4(0.65)$ & $0.05(0.21)$ & $<0.05$ \\
\hline Learning problems & $1.9(0.57)$ & $0.21(0.34)$ & $<0.05$ \\
\hline Psychosomatic & $0.56(0.34)$ & $0.53(0.41)$ & 0.452 \\
\hline Anxiety & $0.42(0.32)$ & $0.51(0.35)$ & 0.523 \\
\hline
\end{tabular}


A

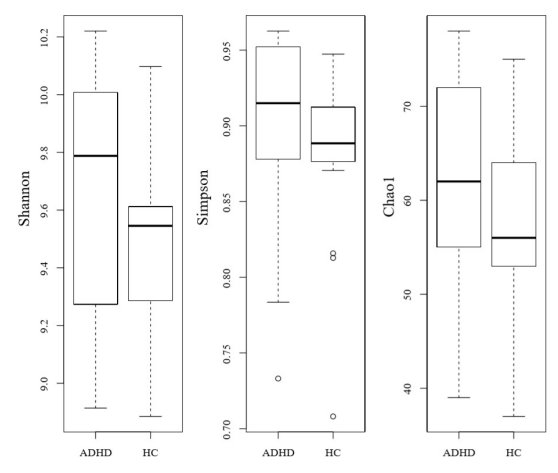

B

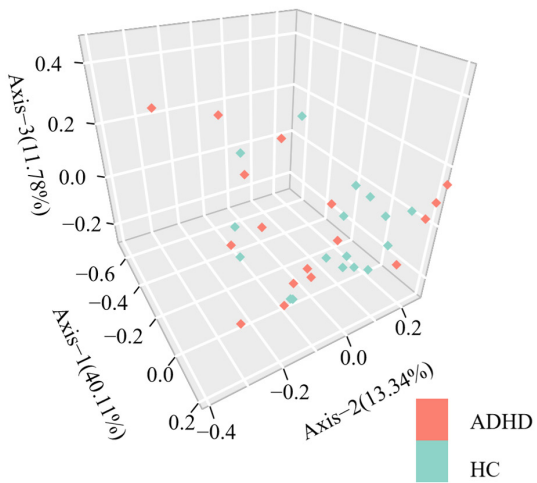

C

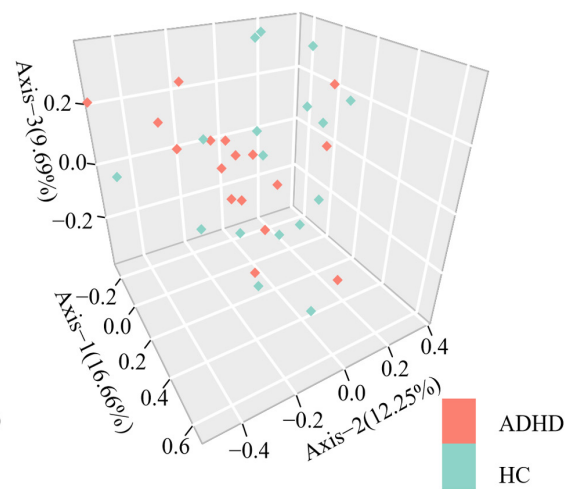

FIGURE 1 | (A) Comparison of alpha diversity indexes between the ADHD and HC groups. (B) Permutational multivariate analysis of variance (PERMANOVA) of microbial communities of the participants at the genus level. (C) PERMANOVA of microbial communities of the participants at the species level.

A

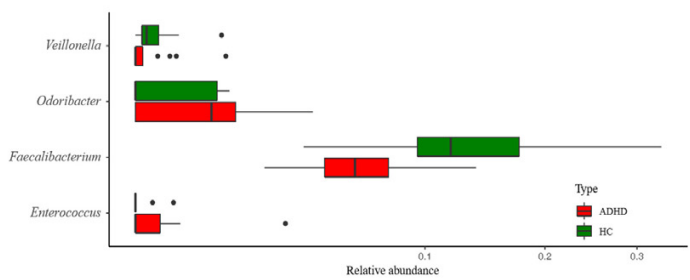

C

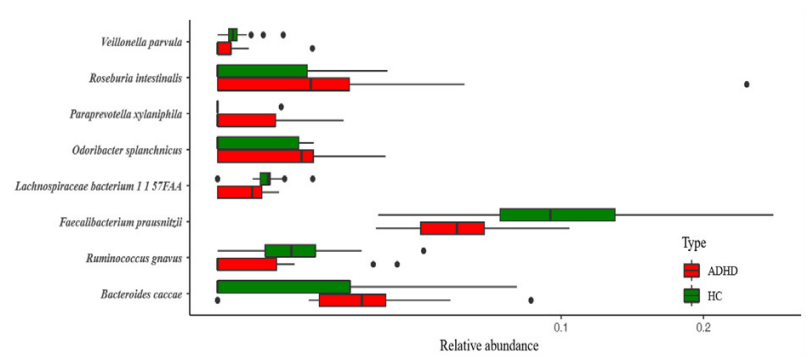

B

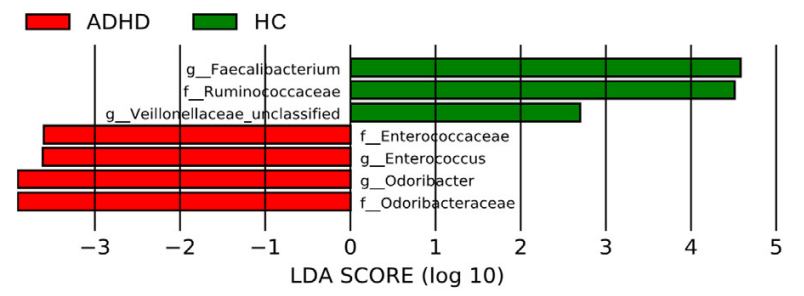

D

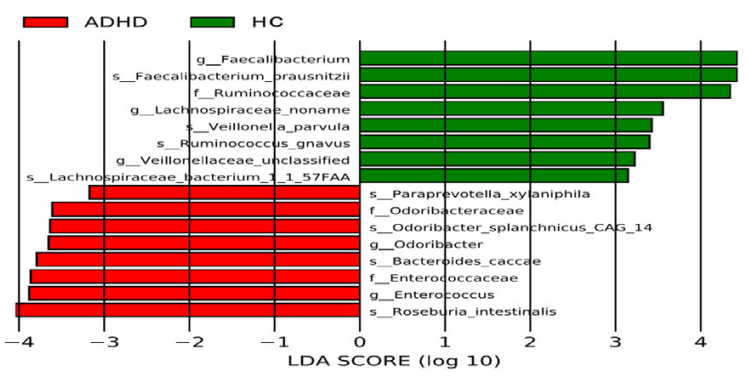

FIGURE 2 | Comparison of different bacteria at the genus and species level between the ADHD and HC groups. (A) Wilcoxon test result at the genus level $(P<0.05)$. (B) LEfSe result at the genus level (LDA > 2). (C) Wilcoxon test result at the species level $(P<0.05)$. (D) LEfSe result at the species level (LDA $>2)$.

Enterococcaceae were significantly increased in the ADHD group, while Ruminococcaceae was significantly decreased (LDA $>$ 2, Figure 2D).

\section{KEGG Analysis of Metabolism}

A total of 6294 KEGG Orthology (KO) terms were used to annotate the genes. Wilcoxon tests showed $91 \mathrm{KOs}$ that were significantly different between the two groups $(P<0.01$, Figure 3). These included terms related to the neurotransmitter dopamine; the genes encoding the catalytic subunit of protein phosphatase-1 (PP1), threonine synthase, and 6-pyruvoyl5,6,7,8-tetrahydropterin were significantly upregulated in the ADHD group, while the gene encoding 4-hydroxy threonine4-phosphate dehydrogenase was significantly downregulated $(P<0.05$, Figure 4).

\section{DISCUSSION}

The mammalian intestinal tract contains more than 100 trillion microorganisms; as the largest ecosystem in the body, it influences host physiological functions (Agus et al., 2018). The brain-gut axis theory proposes that there is a bidirectional regulatory mechanism between the intestinal flora and the brain. Children with ADHD may have abnormal neurotransmission, and the intestinal flora may regulate the level of neurotransmitters via complex neuroendocrine pathways (Richarte et al., 2018). A systematic review revealed two studies that assessed the correlation between ADHD and intestinal flora (Lacorte et al., 2019). Both employed 16S rRNAsequencing technology and only analyzed the difference in gut microflora (Jiang et al., 2018; Prehn-Kristensen et al., 2018). 


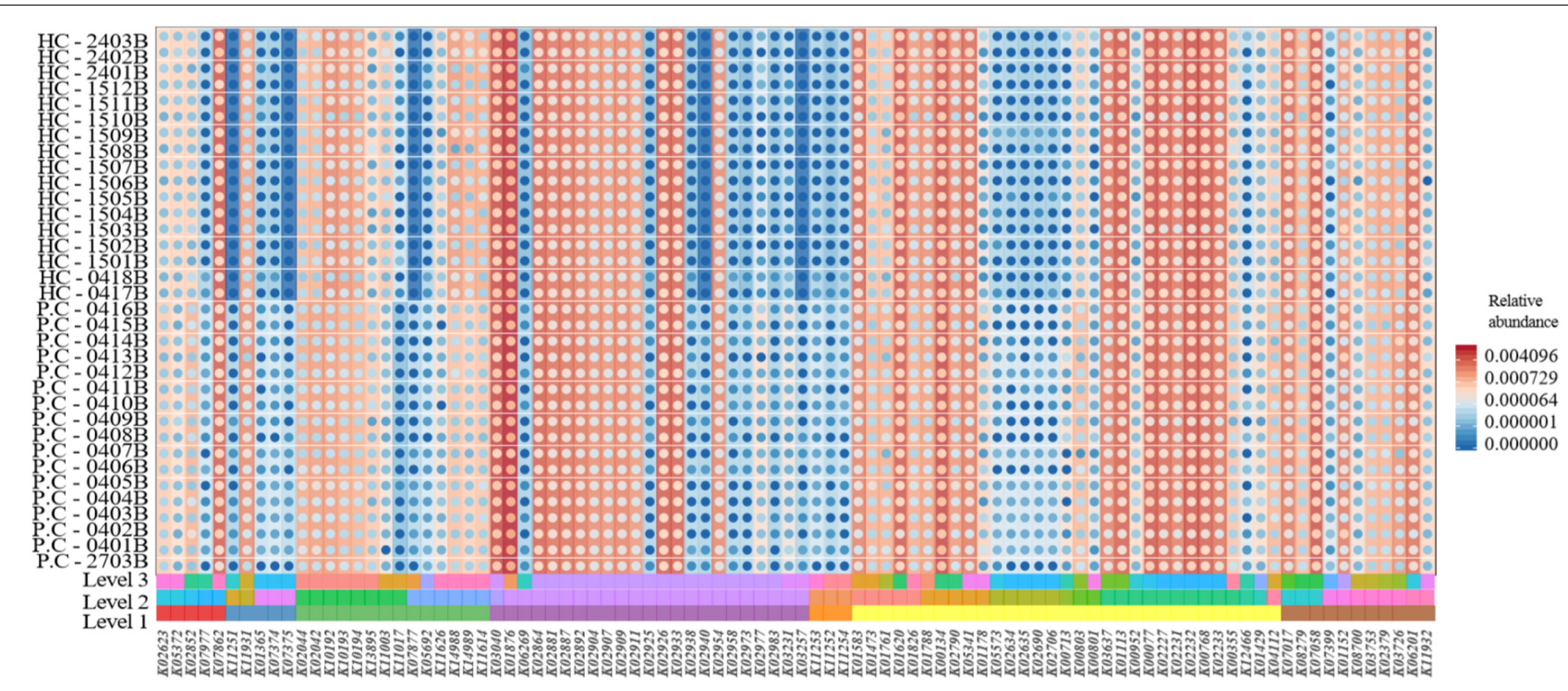

FIGURE 3 | Comparison of Kyoto Encyclopedia of Genes and Genomes (KEGG) functional annotations between the ADHD and HC groups.

We also applied shotgun metagenomic sequencing to the whole genomes of microorganisms for each specimen, and KEGG was used to analyze the metabolic pathways and identify possible pathogenetic mechanisms.

Similar to earlier reports (Jiang et al., 2018; Prehn-Kristensen et al., 2018), we found obvious differences in the gut microbiota of the ADHD and HC groups. Contrary to one study (PrehnKristensen et al., 2018), we found the alpha diversity of intestinal flora was not significantly different between groups, but subjects with ADHD had significantly lower levels of Faecalibacterium. However, unlike a previous report (Jiang et al., 2018), we also found that the ADHD group had significant decreases in Veillonellaceae, while Enterococcus and Odoribacter were significantly increased. At the species level, $F$. prausnitzii, L. bacterium, and $R$. gnavus were significantly reduced in the ADHD group, while B. caccae, O. splanchnicus, $P$. xylaniphila, and $V$. parvula were significantly increased. Additionally, we found that children with ADHD were more prone to constipation; consistent with our finding, previous studies have reported that the imbalance of intestinal flora is closely related to the occurrence of constipation (Huang et al., 2018; Wen et al., 2018; Wang L. et al., 2019).

The pathogenesis of ADHD remains unclear. One research group reported that abnormal levels of neurotransmitters are involved in the disease process (Kovács et al., 2019). Based on this theory, central nervous system (CNS) stimulants such as methylphenidate hydrochloride are widely used as first-line treatments for ADHD. The mechanism may involve inhibition of presynaptic reuptake of noradrenaline and dopamine; higher synaptic levels of these neurotransmitters may help to control symptoms, but clinical treatment effects vary among patients (Wigal et al., 2017).

Previous studies have shown that the gut microbiota could affect the brain-gut axis and contribute to the pathogenesis of neurological diseases including Parkinson's, epilepsy, autism spectrum disorders, and tic disorders (Zhao et al., 2017; Kovács et al., 2019; Rude et al., 2019). Early intestinal flora establishment can affect nervous system development, resulting in anxiety behaviors and other mental health problems after maturity, and treatment of pregnant female rats with lowdose antibiotics has been shown to lead to an imbalance of intestinal flora, with subsequent alterations in the behavior of offspring (Borre et al., 2014; Leclercq et al., 2017; Zhao et al., 2017). In another study where gut bacteria from patients with schizophrenia were transplanted into germ-free mice, the mice developed psychotic symptoms due to altered regulation of the glutamine-glutamate-gamma-aminobutyric acid pathway by the transplanted gut bacteria (Zheng et al., 2019). Similarly, transplantation of intestinal flora from patients with Parkinson's disease into a germ-free Parkinson's disease mouse model significantly increased motor symptoms of the mice, which were ameliorated by antibiotic treatment (Sampson et al., 2016).

According to the results of our experiment, we speculated that the abnormality of intestinal flora might be one of the bases of the onset of ADHD, combined with previous studies, we proposed the following conjecture about its mechanism of action. In this study, children with $\mathrm{ADHD}$ exhibited a reduction of Faecalibacterium. This has been observed in both animal and human studies and has been implicated in various allergic diseases such as asthma, eczema, and allergic rhinitis (Penders et al., 2007; Arrieta et al., 2015; Melli et al., 2016). In clinical practice, atopic children have a $30-50 \%$ increased risk of ADHD (Schans et al., 2017). We therefore speculate that the reduction of this bacterial genus may generate allergies via the brain-gut axis by affecting neurotransmitter release and inducing the pathogenesis of ADHD. One study reported that $\mathrm{ADHD}$ was more likely to be induced by diets high in fat, protein, and sugar, which also decrease Faecalibacterium 


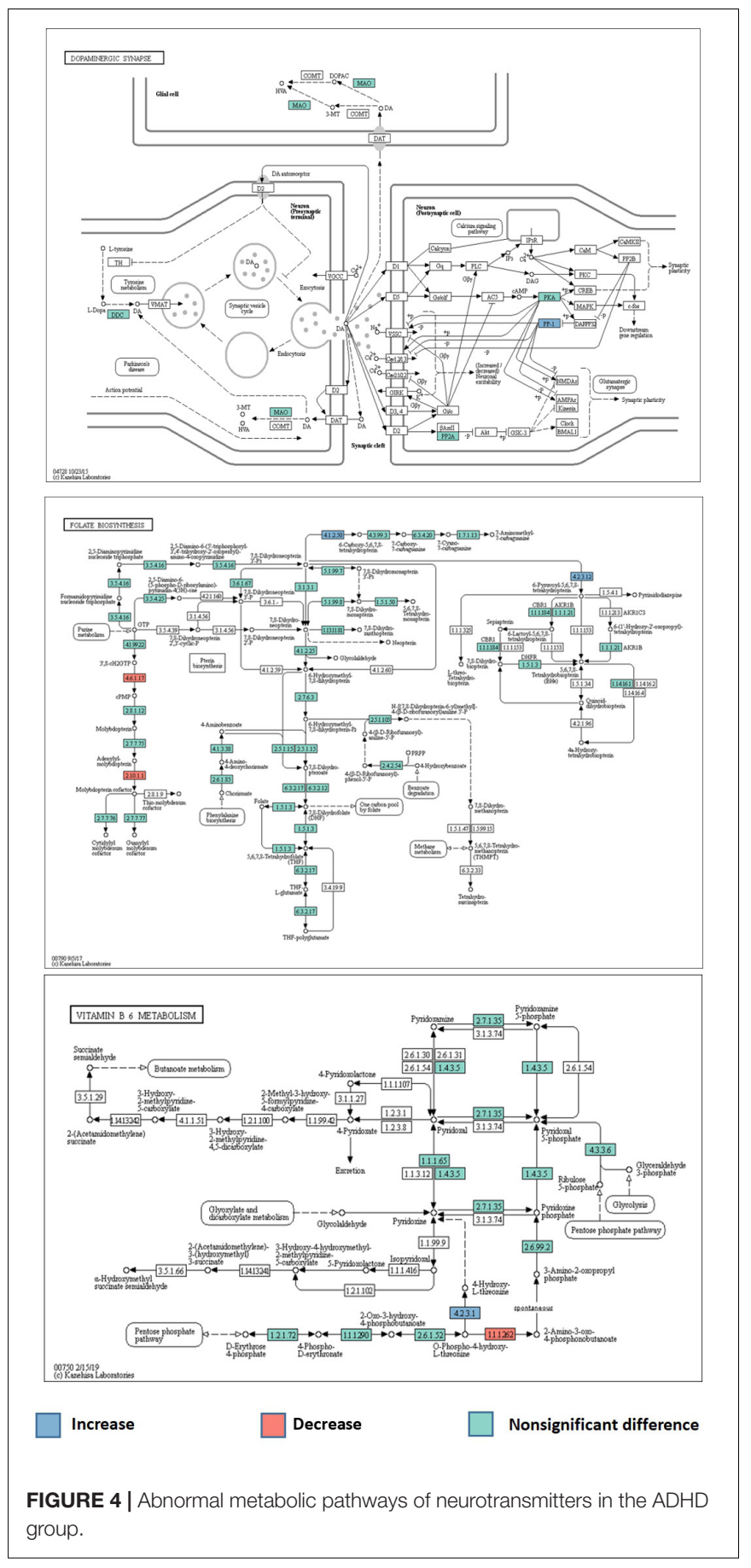

levels (Howard et al., 2011). Faecalibacterium may exert antiinflammatory effects, and the abnormal levels may lead to higher expression of inflammatory factors (Qiu et al., 2013; Quévrain et al., 2016). Notably, children with ADHD have significantly higher levels of inflammatory cytokines than normal children (Mitchell and Goldstein, 2014). Inflammatory cytokines can cross the blood-brain barrier (BBB) and affect nervous system development and brain function (Wong et al., 2016). We therefore hypothesized that Faecalibacterium dysregulation may cause changes in inflammatory cytokine levels and participate in ADHD pathogenesis.

We also found that the proportion of Enterococcus was significantly increased in the ADHD group, and Enterococcus has been reported to be closely related to neurotransmitter release. One study demonstrated that Enterococcus abundance is significantly increased in mice lacking the $5-\mathrm{HT}$ transporter (Singhal et al., 2019); deficiency of this transporter can lead to decreased 5-HT levels, which is related to ADHD onset (Wang et al., 2018). Interestingly, a study showed Enterococcus could lead to excessive intestinal conversion of levodopa (the first-line treatment for Parkinson's disease) into dopamine, however, peripheral dopamine cannot penetrate the BBB to enter the CNS, thus reducing the effectiveness of levodopa (Maini Rekdal et al., 2019). Furthermore, the abnormal increase in Enterococcus could also cause excessive activation of tyrosine decarboxylase, which increases the decarboxylation of tyrosine and phenylalanine in the gastrointestinal tract, leading to decreased levels in the CNS and subsequent low levels of levodopa (the drug precursor of dopamine) (Maini Rekdal et al., 2019). Both of these pathways can affect the concentration of dopamine in the CNS, which may aggravate Parkinson's symptoms (Maini Rekdal et al., 2019). Previous studies have shown that ADHD onset is related to decreased CNS levels of dopamine (Roncero and Álvarez, 2014; Ledonne and Mercuri, 2017). As above, we speculate that the observed increase in Enterococcus may lower intracranial dopamine and contribute to the development of ADHD. In addition, our observation of a higher proportion of Odoribacter in subjects with ADHD is similar to the results of a previous study that found higher Odoribacter levels in individuals with pediatric acute-onset neuropsychiatric syndrome (PAN) and pediatric autoimmune neuropsychiatric disorders associated with streptococcal infections (PANDAS) (Quagliariello et al., 2018). Additionally, Phylogenetic Investigation of Communities by Reconstruction of Unobserved States (PICRUSt) analysis of this study showed that the dopamine metabolic pathway was significantly reduced in PAN and PANDAS (Quagliariello et al., 2018). Odoribacter may cause abnormalities in dopamine metabolism that contribute to ADHD. Previous studies (Quagliariello et al., 2018; Maini Rekdal et al., 2019) found that abnormal Enterococcus and Odoribacter levels were associated with dysregulated neurotransmitter production. Abnormal levels of these bacteria were also found in our study, suggesting a role in the development of ADHD.

Finally, we performed KEGG analysis to determine the gene functional annotations and abnormalities in metabolic pathways, to verify the speculation of the role of gut microbiota in the pathogenesis of ADHD. Reduced dopamine levels in the CNS may contribute to ADHD pathogenesis. We identified differences in the dopaminergic synaptic pathways between the ADHD and HC groups; the gene encoding PP1 catalytic subunit was significantly upregulated, which was considered to increase synaptic sodium ion flux. Dopamine receptors are transmembrane sodium/chloride-dependent transporters that belong to the family of transporters of norepinephrine, 5HT, and dopamine, and are referred to as neurotransmitter: sodium symporters (NSS) (Navratna and Gouaux, 2019). 
Prolonged sodium-related signal transduction results in the excessive activation of NSS. Metabolic pathway alterations may cause abnormal neurotransmitter transport and reduce their concentrations in the CNS, which could contribute to ADHD. Numerous studies have reported that vitamin B6 plays a key role in nervous system development and neurotransmitter production. A randomized controlled trial of 216 children with ADHD and 216 healthy children found lower vitamin B6 levels in children with ADHD (Wang L.J. et al., 2019). In line with this finding, KEGG analysis indicated abnormalities in the metabolic pathway of vitamin B6 in the ADHD group. The genes encoding 4-hydroxy threonine-4-phosphate dehydrogenase and threonine synthase were significantly downregulated and upregulated, respectively, which could lead to abnormal levels of pyridoxal $5^{\prime}$-phosphate, which is an important coenzyme of aromatic amino acid decarboxylase (AADC) (Montioli et al., 2019). AADC is a key enzyme of dopamine metabolism that converts levodopa into dopamine in the CNS (Baek et al., 2018). A decrease in its activity could lead to the reduction of dopamine concentrations, which could contribute to ADHD onset. In the folate metabolic pathway, a significant upregulation of the gene encoding 6-pyruvoyl-5,6,7,8-tetrahydropterin could promote the generation of tetrahydrobiopterin ( $\mathrm{BH} 4)$. However, tryptophan hydroxylase is a rate-limiting enzyme that catalyzes 5-HT synthesis, with oxygen and $\mathrm{BH} 4$ as substrates (Opladen et al., 2016; Scotton et al., 2019). Upregulation of the gene encoding 6-pyruvoyl-5,6,7,8-tetrahydropterin may lead to the conversion of excessive tryptophan into 5-HT in the intestinal tract, and 5-HT has difficulty crossing the BBB, resulting in decreased CNS 5-HT concentrations, which may contribute to ADHD.

There are several limitations to this study. First, our sample size was relatively small. Second, we did not perform transplantation of intestinal flora to confirm that gut microbiota composition affects ADHD symptoms.

\section{CONCLUSION}

In summary, our results demonstrate that gut microbiota alterations occur in children with ADHD, which may contribute to abnormal metabolism of neurotransmitters. We cautiously speculated that the abnormal intestinal flora might be one of contributing factors of $\mathrm{ADHD}$, the underlying mechanism may be related to changes in microbial functions that affect the

\section{REFERENCES}

Abramov, D. M., Cunha, C. Q., Galhanone, P. R., Alvin, R. J., de Oliveira, A. M., and Lazarev, V. V. (2019). Neurophysiological and behavioral correlates of alertness impairment and compensatory processes in ADHD evidenced by the attention network test. PLoS One 14:e0219472. doi: 10.1371/journal.pone.0219472

Agus, A., Planchais, J., and Sokol, H. (2018). Gut microbiota regulation of tryptophan metabolism in health and disease. Cell Host Microbe 23, 716-724. doi: 10.1016/j.chom.2018.05.003

Arrieta, M. C., Stiemsma, L. T., Dimitriu, P. A., Thorson, L., Russell, S., YuristDoutsch, S., et al. (2015). Early infancy microbial and metabolic alterations function of the neuroendocrine system, leading to reduced levels of 5-HT and dopamine in the CNS, and ultimately to ADHD. Further studies should be carried out to investigate the CNS levels of dopamine and 5-HT, and animal studies are needed for functional verification.

\section{DATA AVAILABILITY STATEMENT}

The raw data supporting the conclusions of this article will be made available by the authors, without undue reservation, to any qualified researcher.

\section{ETHICS STATEMENT}

The studies involving human participants were reviewed and approved by the PLA General Hospital Ethics Committee. Written informed consent to participate in this study was provided by the participants' legal guardian/next of kin.

\section{AUTHOR CONTRIBUTIONS}

LW and W-RG contributed equally to the manuscript. LW and GY contributed to the study conception and design. W-RG, SZ, Y-LS, BW, and LW organized the database. W-RG, Y-LS, SZ, LW, and GY performed the statistical analysis. W-RG, LW, and GY wrote the first draft of the manuscript. All authors wrote sections of the manuscript, contributed to the manuscript revision, and read and approved the submitted version.

\section{FUNDING}

This work was funded by the National Natural Science Foundation of China (reference number 81671279) and National Key Research and Development Project (2018YFC1002500).

\section{ACKNOWLEDGMENTS}

We would like to thank the medical staff at the First Medical Center of the PLA General Hospital for their assistance. We would also like to thank Aegicare (Shenzhen) Technology Co., Ltd., for providing strong technical support for this study.

affect risk of childhood asthma. Sci. Transl. Med. 7:307ra152. doi: 10.1126/ scitranslmed.aab2271

Baek, J. S., Tee, J. K., Pang, Y. Y., Tan, E. Y., Lim, K. L., Ho, H. K., et al. (2018). Improved bioavailability of levodopa using floatable spray-coated microcapsules for the management of Parkinson's disease. Neuromolecular Med. 20, 262-270. doi: 10.1007/s12017-018-8491-0

Banerjee, E., and Nandagopal, K. (2015). Does serotonin deficit mediate susceptibility to ADHD? Neurochem. Int. 82, 52-68. doi: 10.1016/j.neuint.2015. 02.001

Bermúdez-Humarán, L. G., Salinas, E., Ortiz, G. G., Ramirez-Jirano, L. J., Morales, J. A., and Bitzer-Quintero, O. K. (2019). From probiotics to psychobiotics: 
live beneficial bacteria which act on the brain-gut axis. Nutrients 11:E890. doi: $10.3390 /$ nu11040890

Bolger, A. M., Lohse, M., and Usadel, B. (2014). Trimmomatic: a flexible trimmer for Illumina sequence data. Bioinformatics 30, 2114-2120. doi: 10. 1093/bioinformatics/btu170

Borre, Y. E., O'Keeffe, G. W., Clarke, G., Stanton, C., Dinan, T. G., and Cryan, J. F. (2014). Microbiota and neurodevelopmental windows: implications for brain disorders. Trends Mol. Med. 20, 509-518. doi: 10.1016/j.molmed.2014.05.002

Fan, Y., Wang, H., Liu, X., Zhang, J., and Liu, G. (2019). Crosstalk between the ketogenic diet and epilepsy: from the perspective of gut microbiota. Mediators Inflamm. 2019:8373060. doi: 10.1155/2019/8373060

Franzosa, E. A., McIver, L. J., Rahnavard, G., Thompson, L. R., Schirmer, M., Weingart, G., et al. (2018). Species-level functional profiling of metagenomes and metatranscriptomes. Nat. Methods 15, 962-968. doi: 10.1038/s41592-018$0176-\mathrm{y}$

Howard, A. L., Robinson, M., Smith, G. J., Ambrosini, G. L., Piek, J. P., and Oddy, W. H. (2011). ADHD is associated with a "Western" dietary pattern in adolescents. J. Atten. Disord. 15, 403-411. doi: 10.1177/1087054710365990

Huang, L., Zhu, Q., Qu, X., and Qin, H. (2018). Microbial treatment in chronic constipation. Sci. China Life Sci. 61, 744-752. doi: 10.1007/s11427-017-9220-7

Jiang, H. Y., Zhou, Y. Y., Zhou, G. L., Li, Y. C., Yuan, J., Li, X. H., et al. (2018). Gut microbiota profiles in treatment-naïve children with attention deficit hyperactivity disorder. Behav. Brain Res. 347, 408-413. doi: 10.1016/j.bbr.2018. 03.036

Karmakar, A., Goswami, R., Saha, T., Maitra, S., Roychowdhury, A., Panda, C. K., et al. (2017). Pilot study indicate role of preferentially transmitted monoamine oxidase gene variants in behavioral problems of male ADHD probands. BMC Med. Genet. 18:109. doi: 10.1186/s12881-017-0469-5

Khalil, M., Zhang, Z., and Engel, M. A. (2019). Neuro-immune networks in gastrointestinal disorders. Visc. Med. 35, 52-60. doi: 10.1159/000496838

Kim, J. I., Yoo, J. H., Kim, D., Jeong, B., and Kim, B. N. (2018). The effects of GRIN2B and DRD4 gene variants on local functional connectivity in attentiondeficit/hyperactivity disorder. Brain Imaging Behav. 12, 247-257. doi: 10.1007/ s11682-017-9690-2

Kovács, Z., D’Agostino, D. P., Diamond, D., Kindy, M. S., Rogers, C., and Ari, C. (2019). Therapeutic potential of exogenous ketone supplement induced ketosis in the treatment of psychiatric disorders: review of current literature. Front. Psychiatry 10:363. doi: 10.3389/fpsyt.2019.00363

Lacorte, E., Gervasi, G., Bacigalupo, I., Vanacore, N., Raucci, U., and Parisi, P. (2019). A systematic review of the microbiome in children with neurodevelopmental disorders. Front. Neurol. 10:727. doi: 10.3389/fneur.2019. 00727

Langmead, B., and Salzberg, S. L. (2012). Fast gapped-read alignment with bowtie 2. Nat. Methods 9, 357-359. doi: 10.1038/nmeth.1923

Leclercq, S., Mian, F. M., Stanisz, A. M., Bindels, L. B., Cambier, E., Ben-Amram, H., et al. (2017). Low-dose penicillin in early life induces long-term changes in murine gut microbiota, brain cytokines and behavior. Nat. Commun. 8:15062. doi: 10.1038/ncomms 15062

Ledonne, A., and Mercuri, N. B. (2017). Current concepts on the physiopathological relevance of dopaminergic receptors. Front. Cell. Neurosci. 11:27. doi: 10.3389 /fncel.2017.00027

Li, J., Jia, H., Cai, X., Zhong, H., Feng, Q., Sunagawa, S., et al. (2014). An integrated catalog of reference genes in the human gut microbiome. Nat. Biotechnol. 32, 834-841. doi: 10.1038/nbt.2942

Magula, L., Moxley, K., and Lachman, A. (2019). Iron deficiency in South African children and adolescents with attention deficit hyperactivity disorder. J. Child Adolesc. Ment. Health 31, 85-92. doi: 10.2989/17280583.2019.1637345

Maini Rekdal, V., Bess, E. N., Bisanz, J. E., Turnbaugh, P. J., and Balskus, E. P. (2019). Discovery and inhibition of an interspecies gut bacterial pathway for Levodopa metabolism. Science 364:eaau6323. doi: 10.1126/science.aau6323

Melli, L. C., do Carmo-Rodrigues, M. S., Araújo-Filho, H. B., Solé, D., and de Morais, M. B. (2016). Intestinal microbiota and allergic diseases: a systematic review. Allergol. Immunopathol. 44, 177-188. doi: 10.1016/j.aller.2015.01.013

Mitchell, R. H., and Goldstein, B. I. (2014). Inflammation in children and adolescents with neuropsychiatric disorders: a systematic review. J. Am. Acad. Child Adolesc. Psychiatry 53, 274-296. doi: 10.1016/j.jaac.2013.11.013

Montioli, R., Battini, R., Paiardini, A., Tolve, M., Bertoldi, M., Carducci, C., et al. (2019). A novel compound heterozygous genotype associated with aromatic amino acid decarboxylase deficiency: clinical aspects and biochemical studies. Mol. Genet. Metab. 127, 132-137. doi: 10.1016/j.ymgme.2019.05.004

Navratna, V., and Gouaux, E. (2019). Insights into the mechanism and pharmacology of neurotransmitter sodium symporters. Curr. Opin. Struct. Biol. 54, 161-170. doi: 10.1016/j.sbi.2019.03.011

Ng, R., Heinrich, K., and Hodges, E. (2019). Associations between ADHD subtype symptomatology and social functioning in children with ADHD, autism spectrum disorder, and comorbid diagnosis: utility of diagnostic tools in treatment considerations. J. Atten. Disord. doi: 10.1177/1087054719855680 [Epub ahead of print].

Opladen, T., Cortès-Saladelafont, E., Mastrangelo, M., Horvath, G., Pons, R., Lopez-Laso, E., et al. (2016). The international working group on neurotransmitter related disorders (iNTD): a worldwide research project focused on primary and secondary neurotransmitter disorders. Mol. Genet. Metab. Rep. 9, 61-66. doi: 10.1016/j.ymgmr.2016.09.006

Penders, J., Stobberingh, E. E., van den Brandt, P. A., and Thijs, C. (2007). The role of the intestinal microbiota in the development of atopic disorders. Allergy 62, 1223-1236. doi: 10.1111/j.1398-9995.2007.01462.x

Prehn-Kristensen, A., Zimmermann, A., Tittmann, L., Lieb, W., Schreiber, S., Baving, L., et al. (2018). Reduced microbiome alpha diversity in young patients with ADHD. PLoS One 13:e0200728. doi: 10.1371/journal.pone.0200728

Qiu, X., Zhang, M., Yang, X., Hong, N., and Yu, C. (2013). Faecalibacterium prausnitzii upregulates regulatory $\mathrm{T}$ cells and anti-inflammatory cytokines in treating TNBS-induced colitis. J. Crohns Colitis 7, e558-e568. doi: 10.1016/j. crohns.2013.04.002

Quagliariello, A., Del Chierico, F., Russo, A., Reddel, S., Conte, G., Lopetuso, L. R., et al. (2018). Gut microbiota profiling and gut-brain crosstalk in children affected by pediatric acute-onset neuropsychiatric syndrome and pediatric autoimmune neuropsychiatric disorders associated with streptococcal infections. Front. Microbiol. 9:675. doi: 10.3389/fmicb.2018.00675

Quévrain, E., Maubert, M. A., Michon, C., Chain, F., Marquant, R., Tailhades, J., et al. (2016). Identification of an anti-inflammatory protein from Faecalibacterium prausnitzii, a commensal bacterium deficient in Crohn's disease. Gut 65, 415-425. doi: 10.1136/gutjnl-2014-307649

Richarte, V., Rosales, K., Corrales, M., Bellina, M., Fadeuilhe, C., Calvo, E., et al. (2018). [The gut-brain axis in attention deficit hyperactivity disorder: the role of the microbiota]. Rev. Neurol. 66, S109-S114.

Roncero, C., and Álvarez, F. J. (2014). The use of lisdexamfetamine dimesylate for the treatment of ADHD and other psychiatric disorders. Expert Rev. Neurother. 14, 849-865. doi: 10.1586/14737175.2014.932691

Rude, K. M., Pusceddu, M. M., Keogh, C. E., Sladek, J. A., Rabasa, G., Miller, E. N., et al. (2019). Developmental exposure to polychlorinated biphenyls (PCBs) in the maternal diet causes host-microbe defects in weanling offspring mice. Environ. Pollut. 253, 708-721. doi: 10.1016/j.envpol.2019.07.066

Salah, M., Azab, M., Ramadan, A., and Hanora, A. (2019). New insights on obesity and diabetes from gut microbiome alterations in Egyptian adults. OMICS 23, 477-485. doi: 10.1089/omi.2019.0063

Sampson, T. R., Debelius, J. W., Thron, T., Janssen, S., Shastri, G. G., Ilhan, Z. E., et al. (2016). Gut microbiota regulate motor deficits and neuroinflammation in a model of Parkinson's disease. Cell 167, 1469-1480.e12. doi: 10.1016/j.cell.2016. 11.018

Schans, J. V., Çiçek, R., de Vries, T. W., Hak, E., and Hoekstra, P. J. (2017). Association of atopic diseases and attention-deficit/hyperactivity disorder: a systematic review and meta-analyses. Neurosci. Biobehav. Rev. 74( $\mathrm{Pt} \mathrm{A})$, 139-148. doi: 10.1016/j.neubiorev.2017.01.011

Scotton, W. J., Hill, L. J., Williams, A. C., and Barnes, N. M. (2019). Serotonin syndrome: pathophysiology, clinical features, management, and potential future directions. Int. J. Tryptophan Res. 12:1178646919873925. doi: 10.1177/ 1178646919873925

Singhal, M., Turturice, B. A., Manzella, C. R., Ranjan, R., Metwally, A. A., Theorell, J., et al. (2019). Serotonin transporter deficiency is associated with dysbiosis and changes in metabolic function of the mouse intestinal microbiome. Sci. Rep. 9:2138. doi: 10.1038/s41598-019-38489-8

Stewart, A., Davis, G. L., Gresch, P. J., Katamish, R. M., Peart, R., Rabil, M. J., et al. (2019). Serotonin transporter inhibition and 5-HT2C receptor activation drive loss of cocaine-induced locomotor activation in DAT Val559 mice. Neuropsychopharmacology 44, 994-1006. doi: 10.1038/s41386-0180301-8 
Suzuki, C., Ikeda, Y., Tateno, A., Okubo, Y., Fukayama, H., and Suzuki, H. (2019). Acute atomoxetine selectively modulates encoding of reward value in ventral medial prefrontal cortex. J. Nippon Med. Sch. 86, 98-107. doi: 10.1272/jnms. JNMS.2019_86-205

Wang, L., Chen, C., Cui, S., Lee, Y. K., Wang, G., Zhao, J., et al. (2019). Adhesive Bifidobacterium induced changes in cecal microbiome alleviated constipation in mice. Front. Microbiol. 10:1721. doi: 10.3389/fmicb.2019.01721

Wang, L. J., Yu, Y. H., Fu, M. L., Yeh, W. T., Hsu, J. L., Yang, Y. H., et al. (2018). Attention deficit-hyperactivity disorder is associated with allergic symptoms and low levels of hemoglobin and serotonin. Sci. Rep. 8:10229. doi: 10.1038/ s41598-018-28702-5

Wang, L. J., Yu, Y. H., Fu, M. L., Yeh, W. T., Hsu, J. L., Yang, Y. H., et al. (2019). Dietary profiles, nutritional biochemistry status, and attentiondeficit/hyperactivity disorder: path analysis for a case-control study. J. Clin. Med. 8:E709. doi: 10.3390/jcm8050709

Wen, W., Zhang, H., Shen, J., Wei, L., and Shen, S. (2018). Fecal microbiota transplantation for patients with irritable bowel syndrome: a meta-analysis protocol. Medicine 97:e12661. doi: 10.1097/MD.0000000000012661

Wigal, S. B., Childress, A., Berry, S. A., Belden, H., Walters, F., Chappell, P., et al. (2017). Efficacy and safety of a chewable methylphenidate extended-release tablet in children with attention-deficit/hyperactivity disorder. J. Child Adolesc. Psychopharmacol. 27, 690-699. doi: 10.1089/cap.2016.0177
Wong, M. L., Inserra, A., Lewis, M. D., Mastronardi, C. A., Leong, L., Choo, J., et al. (2016). Inflammasome signaling affects anxiety- and depressivelike behavior and gut microbiome composition. Mol. Psychiatry 21, 797-805. doi: 10.1038/mp.2016.46

Zhao, H., Shi, Y., Luo, X., Peng, L., Yang, Y., and Zou, L. (2017). The effect of fecal microbiota transplantation on a child with Tourette syndrome. Case Rep. Med. 2017:6165239. doi: 10.1155/2017/6165239

Zheng, P., Zeng, B., Liu, M., Chen, J., Pan, J., Han, Y., et al. (2019). The gut microbiome from patients with schizophrenia modulates the glutamateglutamine-GABA cycle and schizophrenia-relevant behaviors in mice. Sci. Adv 5:eaau8317. doi: 10.1126/sciadv.aau8317

Conflict of Interest: The authors declare that the research was conducted in the absence of any commercial or financial relationships that could be construed as a potential conflict of interest.

Copyright (C) 2020 Wan, Ge, Zhang, Sun, Wang and Yang. This is an open-access article distributed under the terms of the Creative Commons Attribution License (CC BY). The use, distribution or reproduction in other forums is permitted, provided the original author(s) and the copyright owner(s) are credited and that the original publication in this journal is cited, in accordance with accepted academic practice. No use, distribution or reproduction is permitted which does not comply with these terms. 